\title{
White dwarf accretion and type Ia supernovae
}

\author{
Z. Han and X. Chen \\ Key Laboratory for the Structure and Evolution of Celestial Objects, Yunnan Observatory, \\ Kunming, 650011, China \\ email: zhanwenhan@ynao.ac.cn, cxf@ynao.ac.cn
}

\begin{abstract}
Type Ia supernovae (SNe Ia) are believed to be thermonuclear explosions of carbonoxygen white dwarfs at a mass close to the Chandrasekhar limit. However, a white dwarf at birth has a significantly lower mass and needs to accrete mass to grow to the limit for the explosion. Various progenitor models have been proposed and those models play an important role in our understanding of SNe Ia and cosmology.
\end{abstract}

Keywords. Stars: supernovae, stars: white dwarfs

\section{Introduction}

Type Ia supernovae (SNe Ia) are among the most energetic explosion events in the Universe but exhibit a reamarkable uniformity. They have successfully been used as a cosmological distance indicator to determine $\Omega_{\mathrm{M}}$ and $\Omega_{\Lambda}$ (Riess et al. 1998, Perlmutter et al. 1999), leading to the discovery of the accelerating expansion of the Universe.

SNe Ia are believed to be thermonuclear explosions of carbon-oxygen white dwarfs (CO WDs) of masses close to the Chandrasekhar limit, in which carbon is ignited under degenerate conditions and this result in thermonuclear runaway and then the incineration and complete desctruction of the star.

The masses of CO WDs at birth is not more massive than $\sim 1.1 M_{\odot}$ according to stellar evolutionary theory, and therefore accretion is necessary for CO WDs to grow in mass. The accretion onto WDs are also crucial for many other objects, such as cataclysmic variables (CVs), Canum Venaticorum (AM CVn) stars, supersoft X-ray sources (SSSs), symbiotic stars, etc.

\section{WD accretion}

When hydrogen-rich material is transferred onto the surface of a CO WD, the material accumulates and then get ignited, and hydrogen is converted to helium and helium to carbon and oxygen. If the mass trasfer rate (mass accumulation rate) is too low, e.g. less than $\sim 4 \times 10^{-8} M_{\odot} \mathrm{yr}^{-1}$, hydrogen or helium shell flashes occur (the burning is unstable) and part of the WD masses may be blown away and the WD gets eroded. If the mass transfer rate is too high, higher than a critical rate of $\sim 3 \times 10^{-7} M_{\odot} \mathrm{yr}^{-1}$, the accreting WD would develop an extended envelope and become an AGB-like star. Therefore the mass transfer rate needs to be in a very narrow range to keep the accreted layer burning steadily and the WD growing in mass. Thus WDs have a small probability to evolve to SN Ia explosions. Hachisu et al. (1996), however, proposed an optically thick wind model for the WD accretion as below. If the mass acrretion rate is higher than the critical rate, the accreted matter is burned steadily, the WD grows at the critical rate, and the unprocessed matter is lost from the system in the form of an optically thick wind. If the 
mass accretion rate is lower than one eighth of the critical rate, the WD does not grow. If the mass acrretion rate is in between, the WD grows at the rate. Such a model makes a WD produce a SN Ia more likely than that previously believed (see Fig. 1).

\section{Progenitor models of SNe Ia}

Single degenerate (SD) model and double degenerate (DD) model are currently the two major competing models for the progenitors of SNe Ia, though some other models, including the sub-Chandrasekhar mass model, the super-Chandrasekharmass model, the single star model, the core-degenerate model, etc., have been proposed to explain the observed diversity of SNe Ia (see a detailed review by Wang \& Han, 2012). In the SD model, a CO WD accretes matter from a non-degenerate compnion star, and the companion star (the mass donor) can be a main sequence (MS) star, a red giant (RG) star or a helium star. The WD explodes as a SN Ia when it reaches the Chandrasekhar mass limit (see Fig. 1). In the DD model, a CO WD pair merge due to angular momentum loss via gravitational wave radiation, and may explode as a SN Ia if the total mass exceeds the Chandrasekhar limit.

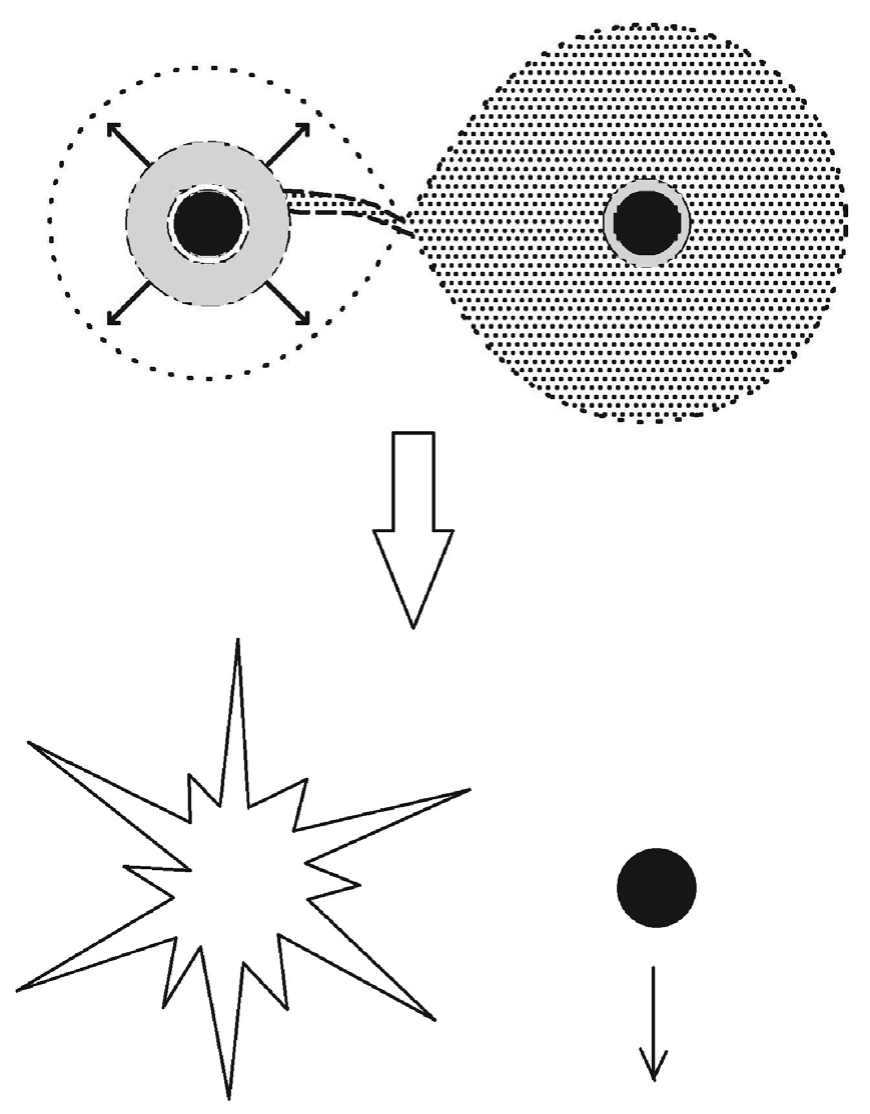

Figure 1. A schematic view of WD accretion and type Ia supernova (SN Ia) explosion. The donor fills its Roche lobe and transfers its hydrogen-rich material to the WD. Part of the hydrogen-rich material accreted is burnt onto the CO WD and the unprocessed material leaves the system in the form of an optically-thick wind. The WD grows in mass till it reaches Chandrasekhar mass limit to explode as a SN Ia. The remnant donor star is left after the explosion and is with a high space velocity. 
The SD model explains the uniformity of SNe Ia naturally as the CO WDs explode at the same mass. Observationally, SNe Ia are found both in spiral galaxies and in elliptical galaxies, which means that the time from star formation to SN explosion (i.e. the delay time) varies greatly. The three channels in the model can in principle explain SNe Ia of various delay times. The helium star donor chanel produces SNe Ia of short delay time $(<0.1 \mathrm{Gyr})$, the MS star donor channel is for intermediate delay time of 0.1 - $1.0 \mathrm{Gyr}$, and the RG donor channel for long delay time ( $>1$ Gyr). Employing Eggleton's stellar evolution code (Eggleton 1973, Han et al. 1994) and Han's binary population synthesis (BPS) approach (Han et al. 1995), many authors (e.g. Han \& Podsiadlowski 2004, Wang et al., 2009, Chen et al.2011, Meng et al. 2009) have studied in detail the three channels. They have obtained the parameter space of WD binaries leading to SN Ia explosions, i.e. a WD binary may evolve to a SN Ia if its WD mass, secondary mass and orbital period is in the parameter space. They have also obtained the birthrates and the delay time distribution. Unlike helium star donor channel and MS star donor channel, where the mass transfer from the donor is very likely to be stable, the RG donor channel suffers from stability problem. The mass tansfer is probably dynamically unstable and leads to the formation of common envelope $(\mathrm{CE})$, and the $\mathrm{CE}$ engulfs the WD and the core of the giant, and such a system would not evolve to a SN Ia. Hachisu et al. (1999) proposed a mass-stripping model, in which part of the giant's envelope is stripped away by stellar wind from WD so that to stablize the mass transfer. Chen \& Li (2007), Xu \& Li (2009), Wang et al. (2010) proposed a disk model for the channel with a low mass giant, in which the disk is responsible for the growth of WD's mass. Chen et al. (2011) proposed a model in which the giant's mass may have been greatly reduced due to tidally enhanced stellar wind before the onset of RLOF, and some mass of wind can be accreted and the RLOF is more likely to be stable. The main difficulties of the SD model is that the birthrate is lower than that inferred observationally, by a factor of 3 to 10, and that weak hydrogen lines should be seen in the spectra of SNe Ia.

The DD model, on the other hand, explains naturally the absence of of hydrogen and helium lines in SN Ia spectra. It also naturally explains the observed delay time distribution $\left(\sim t^{-1}\right)$ as the delay time is dominated by the decay time of the orbit of the DD systems. The merger may have masses from $1.4 M_{\odot}$ to $2.1 M_{\odot}$ and can then result in the diversity of SNe Ia. However, CO WD mergers are more likely to lead to core collapse to produce neutron stars rather than to thermonulclear explosions (Nomoto \& Iben 1985). Recent SPH simulations show, however, that a DD merger may produce a SN Ia if the merger process is violent enough (Pakmor et al. 2012). It was widely believed that the birthrate of SNe Ia from the DD model is consistent with observational ones. However, as pointed out by Chen et al. (2012) and Toonen et al. (2012), this is not true any more due to the following reasons. (1) The merger process of DDs that leads to SN Ia explosion is not conservative as shown by Han \& Webbink (1999); (2) The merger process needs to be violent in order to initiate an explosion as shown by Pakmor et al. (2012) and thus requires a mass ratio of DD not far from unity; (3) The BPS model needs to be calibrated to reproduce DDs observed first before the BPS model can be used to study the birthrate of SNe Ia from the DD model, as shown by Toonen et al. (2012).

\section{Verification of the progenitor models}

The progenitor models play a key role in our understanding of SNe Ia as a cosmological distance indicator and therefore are crucial to the development of precision cosmology. The most straightforward way to identify the progenitor models is to find the remnant companion star of a SN Ia. The donor star is left as a remnant after SN explosion in the 
SD model (see Fig. 1), while no remant star is left in the DD model. The remnant star in the SD model is predicted to have a high space velocity (Han 2008) and therefore Tycho $\mathrm{G}$ could be the remnant star of Tycho's supernova (Ruiz-Lapuente et al. 2004) though its rotational velocity is lower than expected (Han 2008, Pan et al.2012, Liu et al.2012). However, an absence of ex-companion stars in the SN Ia remnant SNR 0509-67.5 may support the DD model.

Many other observations can also be used to constrain the progenitor models of SNe Ia. Those observations include that of the properties of host galaxies, the birthrates, the delay time distributions, the candidate progenitors, the impact-stripped material, gas outflow signature, the wind-blown cavity, the early optical and UV emission, the early radio and X-ray emission, the pre-explosion images, the spectropolarimetry. Observationally, there is increasing evidence supporting that some SNe Ia are from the SD model and some could be from the DD model.

\section{References}

Chen, W. \& Li, X. 2007, ApJ 658, L51

Chen, X., Han, Z., \& Tout, C. A. 2011, ApJ 735, L31

Chen, X., Jeffery, C. S., Zhang, Z., \& Han, Z. 2012, ApJ 755, L9

Eggleton, P. P. 1973, MNRAS, 163, 279

Hachisu, I., Kato, M., \& Nomoto, K. 1996, ApJ, 470, L97

Hachisu, I., Kato, M., \& Nomoto, K. 1999, ApJ, 522, 487

Han, Z. 2008, ApJ, 677, L109

Han, Z. \& Podsiadlowski, Ph. 2004, MNRAS, 350, 1301

Han, Z., Podsiadlowski, Ph., \& Eggleton, P. P. 1994, MNRAS, 270, 121

Han, Z., Podsiadlowski, Ph., \& Eggleton, P. P. 1995, MNRAS, 272, 800

Han, Z., Podsiadlowski, Ph., \& Lynas-Gray, A. E. 2007, MNRAS, 380, 1098

Han, Z. \& Webbink, R. F. 1999, A\&A, 349, L17

Liu, Z., Pakmor, R., \& Roepke, F. K. et al. 2012, arXiv1209.4458

Meng, X., Chen, X., \& Han, Z. 2009, MNRAS, 395, 2103

Nomoto, K. \& Iben, I. Jr. 1985, ApJ, 297, 531

Pakmor, R., Kromer, M., \& Taubenberger, S., et al. 2012, ApJ, 747, L10

Pan, K., Ricker, P., \& Taam, R. 2012, arXiv1270.0170

Perlmutter, S., Aldering, G., \& Goldhaber, G., et al. 1999, ApJ, 517, 565.

Riess, A., Filippenko, A. V., \& Challis, P., et al. 1998, AJ, 116, 1009

Ruiz-Lapuente, P., Comeron, F., \& Mendez, J. et al. 2004, Nature, 431, 1069

Toonen, S., Nelemans, G., \& Portegies Zwart, S. 2012, A\&SA, 546, 70

Wang, B. \& Han, Z. 2012, New Astron. Revs, 56, 122

Wang, B., Li, X., \& Han, Z. 2010, MNRAS, 401, 2729

Wang, B., Meng, X., Chen, X., \& Han, Z. 2009, MNRAS, 395, 847

Xu, X. \& Li, X. 2009, A\&A A 495, 243 